\title{
Chemical Pollutants Adsorbed to Ingested Microbeads from Personal Care Products Accumulate in Fish
}

3

4

5

Peter Wardrop, Jeff Shimeta, Dayanthi Nugegoda, Paul D. Morrison, Ana Miranda, Min Tang \& Bradley O. Clarke

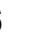

Australia.

8

*Corresponding Author: bradley.clarke@rmit.edu.au;+61 399253358

\section{Supplementary Information}

Table S1. Mean PBDE concentrations of fish feed, control MBs and PBDEs + MBs used for dietary exposure experiment.

Table S2. PBDE concentrations in experiment samples rainbow fish (ng g $\left.\mathrm{g}^{-1} \mathrm{ww}\right)$.

Table S3. Mean PBDE congener concentrations ( $\left.\mathrm{ng} \mathrm{g}^{-1} \mathrm{ww}\right)$ in each treatment.

Figure S1. Micrographs of Murray River rainbow fish faecal matter showing presence of MBs (enclosed with red circles).

Figure S2. Recovery of LCS and MS samples analysed alongside experiment samples (mean $\pm 1 \mathrm{SE}, \mathrm{n}=3$ ). LCS and MS samples were spiked with $10 \mathrm{ng}$ of each target PBDE (100 ng of BDE-209). 
Table S1. Mean PBDE concentrations of fish feed, control MBs and PBDEs + MBs used for dietary exposure experiment.

\begin{tabular}{lccc}
\hline Congener & $\begin{array}{c}\text { Fish Food } \\
\mathbf{n g ~ g}^{-1}(\mathbf{R S D} \%)\end{array}$ & $\begin{array}{c}\text { Control MBs } \\
\mathbf{n g ~ g}^{-1}(\mathbf{R S D} \%)\end{array}$ & $\begin{array}{c}\text { PBDE + MBs } \\
\mathbf{n g ~ g}^{-1} \text { (RSD\%) }\end{array}$ \\
\hline BDE-28 & n.d. & $0.16(13)$ & $192.5(1.4)$ \\
BDE-47 & $0.08(138)$ & n.d. & $168.0(3.2)$ \\
BDE-100 & $0.02(173)$ & n.d. & $178.4(2.8)$ \\
BDE-99 & $0.11(173)$ & n.d. & $189.9(3.8)$ \\
BDE-154 & $0.01(140)$ & $0.02(141)$ & $180.5(3.8)$ \\
BDE-153 & $0.01(98)$ & n.d. & $177.6(5.2)$ \\
BDE-183 & n.d. & $0.11(69)$ & $186.2(6.4)$ \\
BDE-209 & n.d. & n.d. & $1761.4(12.5)$ \\
\hline TOTAL & $0.24(10)$ & $0.28(42)$ & $30344(6)$ \\
\hline n.d., less than detection limit & & &
\end{tabular}


Table S2. PBDE concentrations ( $\mathrm{ng} \mathrm{g}^{-1} \mathrm{ww}$ ) in experiment samples rainbow fish

\begin{tabular}{|c|c|c|c|c|c|c|c|c|c|}
\hline Sample ID & $\begin{array}{c}\text { BDE- } \\
28\end{array}$ & $\begin{array}{c}\text { BDE- } \\
47\end{array}$ & $\begin{array}{c}\text { BDE- } \\
100\end{array}$ & $\begin{array}{c}\text { BDE- } \\
99\end{array}$ & $\begin{array}{c}\text { BDE- } \\
154\end{array}$ & $\begin{array}{c}\text { BDE- } \\
153\end{array}$ & $\begin{array}{c}\text { BDE- } \\
183\end{array}$ & $\begin{array}{c}\text { BDE- } \\
209\end{array}$ & $\sum$ PBDE \\
\hline Day 0-1 & 0.0787 & 0.8658 & 0.1196 & 0.1276 & 0.1397 & 0.0457 & n.d. & n.d. & 1.377 \\
\hline Day 0-2 & 0.0579 & 1.7601 & 0.2376 & 0.0982 & 0.2823 & 0.0697 & n.d. & n.d. & 2.506 \\
\hline $21 \mathrm{~F}-1$ & 0.059 & 1.322 & 0.127 & 0.031 & 0.056 & 0.035 & n.d. & n.d. & 1.629 \\
\hline $21 \mathrm{~F}-2$ & 0.059 & 1.324 & 0.128 & n.d. & 0.054 & 0.038 & n.d. & n.d. & 1.603 \\
\hline $21 \mathrm{~F}-3$ & 0.029 & 0.482 & 0.021 & n.d. & n.d. & 0.039 & n.d. & n.d. & 0.571 \\
\hline $21 \mathrm{~F}-4$ & 0.050 & 1.362 & 0.109 & n.d. & 0.062 & 0.033 & n.d. & n.d. & 1.617 \\
\hline $21 \mathrm{~F}-5$ & 0.129 & 1.465 & 0.142 & n.d. & 0.147 & 0.040 & n.d. & n.d. & 1.922 \\
\hline $21 \mathrm{CP}-1$ & 0.039 & 1.038 & 0.078 & n.d. & 0.050 & 0.035 & n.d. & n.d. & 1.241 \\
\hline 21 CP-2 & 0.033 & 0.286 & 0.020 & n.d. & 0.033 & 0.040 & n.d. & n.d. & 0.412 \\
\hline $21 \mathrm{CP}-3$ & 0.064 & 2.039 & 0.159 & n.d. & n.d. & 0.049 & n.d. & n.d. & 2.310 \\
\hline $21 \mathrm{CP}-4$ & 0.050 & 1.842 & 0.182 & n.d. & 0.044 & 0.054 & n.d. & n.d. & 2.172 \\
\hline $21 \mathrm{CP}-5$ & 0.054 & 1.062 & 0.085 & n.d. & 0.025 & 0.030 & n.d. & n.d. & 1.255 \\
\hline 21 BDE-1 & 1.098 & 2.826 & 0.612 & n.d. & 0.284 & 0.158 & n.d. & n.d. & 4.977 \\
\hline 21 BDE-2 & 1.352 & 2.777 & 0.715 & n.d. & 0.403 & 0.146 & n.d. & n.d. & 5.392 \\
\hline 21 BDE-3 & 1.817 & 3.617 & 0.937 & n.d. & 0.531 & 0.243 & n.d. & n.d. & 7.145 \\
\hline 21 BDE-4 & 0.656 & 1.490 & 0.328 & n.d. & 0.191 & 0.074 & n.d. & n.d. & 2.738 \\
\hline 21 BDE-5 & 1.236 & 2.337 & 0.709 & n.d. & 0.441 & 0.192 & n.d. & n.d. & 4.915 \\
\hline $42 \mathrm{~F}-1$ & 0.060 & 1.112 & 0.105 & n.d. & 0.109 & 0.062 & n.d. & n.d. & 1.448 \\
\hline 42 F-2 & 0.029 & 0.294 & 0.027 & n.d. & 0.052 & 0.027 & n.d. & n.d. & 0.429 \\
\hline 42 F-3 & 0.032 & 0.250 & 0.035 & n.d. & 0.047 & 0.033 & n.d. & n.d. & 0.396 \\
\hline $42 \mathrm{~F}-4$ & 0.027 & 0.199 & 0.018 & n.d. & 0.041 & 0.036 & n.d. & n.d. & 0.321 \\
\hline 42 F-5 & 0.063 & 1.034 & 0.117 & n.d. & 0.052 & 0.041 & n.d. & n.d. & 1.307 \\
\hline 42 CP-1 & 0.060 & 0.324 & 0.060 & n.d. & 0.100 & 0.044 & n.d. & n.d. & 0.588 \\
\hline 42 CP-2 & 0.034 & 0.401 & 0.040 & n.d. & 0.040 & 0.033 & n.d. & n.d. & 0.547 \\
\hline 42 CP-3 & 0.045 & 1.064 & 0.100 & n.d. & 0.061 & 0.032 & n.d. & n.d. & 1.302 \\
\hline 42 CP-4 & 0.037 & 0.586 & 0.127 & 0.433 & 0.051 & 0.082 & n.d. & n.d. & 1.316 \\
\hline 42 CP-5 & 0.065 & 0.915 & 0.087 & n.d. & 0.040 & 0.034 & n.d. & n.d. & 1.141 \\
\hline 42 BDE-1 & 1.824 & 2.813 & 0.997 & n.d. & 0.655 & 0.192 & 0.079 & n.d. & 6.560 \\
\hline 42 BDE-2 & 1.668 & 2.470 & 0.957 & n.d. & 0.549 & 0.196 & 0.071 & n.d. & 5.910 \\
\hline 42 BDE-3 & 1.860 & 3.136 & 1.280 & n.d. & 0.781 & 0.281 & 0.078 & n.d. & 7.416 \\
\hline 42 BDE-4 & 1.804 & 2.114 & 1.096 & 0.021 & 0.617 & 0.251 & 0.054 & n.d. & 5.957 \\
\hline 42 BDE-5 & 1.274 & 1.658 & 0.645 & 0.007 & 0.432 & 0.188 & 0.046 & n.d. & 4.250 \\
\hline $63 \mathrm{~F}-1$ & 0.115 & 1.397 & 0.262 & n.d. & 0.157 & 0.056 & n.d. & n.d. & 1.986 \\
\hline $63 \mathrm{~F}-2$ & 0.075 & 0.529 & 0.059 & n.d. & 0.061 & 0.020 & n.d. & n.d. & 0.744 \\
\hline 63 F-3 & 0.069 & 1.077 & 0.112 & n.d. & 0.040 & 0.015 & n.d. & n.d. & 1.312 \\
\hline 63 F-4 & 0.051 & 0.316 & 0.031 & n.d. & 0.037 & 0.011 & n.d. & n.d. & 0.445 \\
\hline 63 F-5 & 0.091 & 0.513 & 0.055 & n.d. & 0.069 & 0.015 & n.d. & n.d. & 0.743 \\
\hline 63 CP-1 & 0.101 & 0.558 & 0.082 & n.d. & 0.076 & 0.028 & n.d. & n.d. & 0.845 \\
\hline 63 CР-2 & 0.094 & 1.023 & 0.157 & 0.145 & 0.079 & 0.031 & n.d. & n.d. & 1.529 \\
\hline $63 \mathrm{CP}-3$ & 0.152 & 0.639 & 0.151 & n.d. & 0.133 & 0.019 & n.d. & n.d. & 1.094 \\
\hline 63 CP-4 & 0.093 & 0.335 & 0.051 & n.d. & 0.061 & 0.027 & n.d. & n.d. & 0.565 \\
\hline 63 CP-5 & 0.103 & 0.522 & 0.060 & n.d. & 0.092 & 0.021 & n.d. & n.d. & 0.797 \\
\hline 63 BDE-1 & 2.223 & 3.666 & 1.412 & n.d. & 1.333 & 0.273 & 0.041 & n.d. & 8.947 \\
\hline 63 BDE-2 & 2.401 & 3.108 & 2.016 & 0.034 & 1.994 & 0.415 & 0.056 & n.d. & 10.023 \\
\hline 63 BDE-3 & 2.302 & 4.092 & 2.758 & 0.674 & 2.550 & 0.496 & 0.055 & n.d. & 12.927 \\
\hline 63 BDE-4 & 2.366 & 3.199 & 1.740 & 0.070 & 1.324 & 0.385 & 0.036 & n.d. & 9.120 \\
\hline 63 BDE-5 & 1.853 & 2.438 & 1.521 & n.d. & 1.425 & 0.279 & 0.049 & n.d. & 7.564 \\
\hline
\end{tabular}


Table S3. Mean PBDE congener concentrations ( $\mathrm{ng} \mathrm{g}^{-1} \mathrm{ww}$ ) in each treatment.

\begin{tabular}{lccccccccc}
\hline Sample batch & BDE- & BDE- & BDE- & BDE- & BDE- & BDE- & BDE- & BDE- & PBDE \\
& $\mathbf{2 8}$ & $\mathbf{4 7}$ & $\mathbf{1 0 0}$ & $\mathbf{9 9}$ & $\mathbf{1 5 4}$ & $\mathbf{1 5 3}$ & $\mathbf{1 8 3}$ & $\mathbf{2 0 9}$ & \\
\hline Day 0 & 0.07 & 1.31 & 0.18 & 0.11 & 0.21 & 0.06 & n.d. & n.d. & 1.94 \\
21 Day Food & 0.07 & 1.19 & 0.11 & 0.01 & 0.06 & 0.04 & n.d. & n.d. & 1.47 \\
Only & & & & & & & & & \\
$\begin{array}{l}\text { 42 Day Food } \\
\text { Only }\end{array}$ & 0.04 & 0.58 & 0.06 & n.d. & 0.06 & 0.04 & n.d. & n.d. & 0.78 \\
$\begin{array}{l}\text { 63 Day Food } \\
\text { Only }\end{array}$ & 0.08 & 0.77 & 0.1 & n.d. & 0.07 & 0.02 & n.d. & n.d. & 1.05 \\
$\begin{array}{l}\text { 21 Day Clean } \\
\text { MBs }\end{array}$ & 0.05 & 1.25 & 0.1 & n.d. & 0.03 & 0.04 & n.d. & n.d. & 1.48 \\
$\begin{array}{l}\text { 42 Day Clean } \\
\text { MBs }\end{array}$ & 0.05 & 0.66 & 0.08 & 0.09 & 0.06 & 0.04 & n.d. & n.d. & 0.98 \\
$\begin{array}{l}\text { 63 Day Clean } \\
\text { MBs }\end{array}$ & 0.11 & 0.62 & 0.1 & 0.03 & 0.09 & 0.03 & n.d. & n.d. & 0.97 \\
$\begin{array}{l}\text { 21 Day PBDE } \\
\text { MBs }\end{array}$ & 1.23 & 2.61 & 0.66 & n.d. & 0.37 & 0.16 & n.d. & n.d. & 5.03 \\
$\begin{array}{l}\text { 42 Day PBDE } \\
\text { MBs }\end{array}$ & 1.69 & 2.44 & 1 & 0.01 & 0.61 & 0.22 & 0.07 & n.d. & 6.02 \\
$\begin{array}{l}\text { 63 Day PBDE } \\
\text { MBs }\end{array}$ & 2.23 & 3.3 & 1.89 & 0.16 & 1.73 & 0.37 & 0.05 & n.d. & 9.72 \\
\hline
\end{tabular}




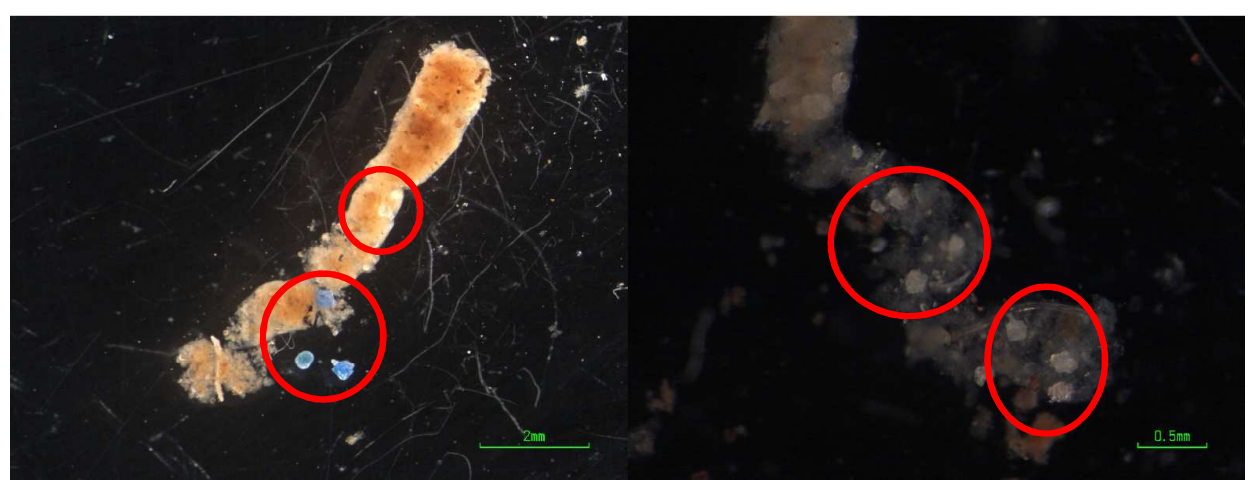

Figure S1. Micrographs of Murray River rainbow fish faecal matter showing presence of MBs (enclosed with red circles). 


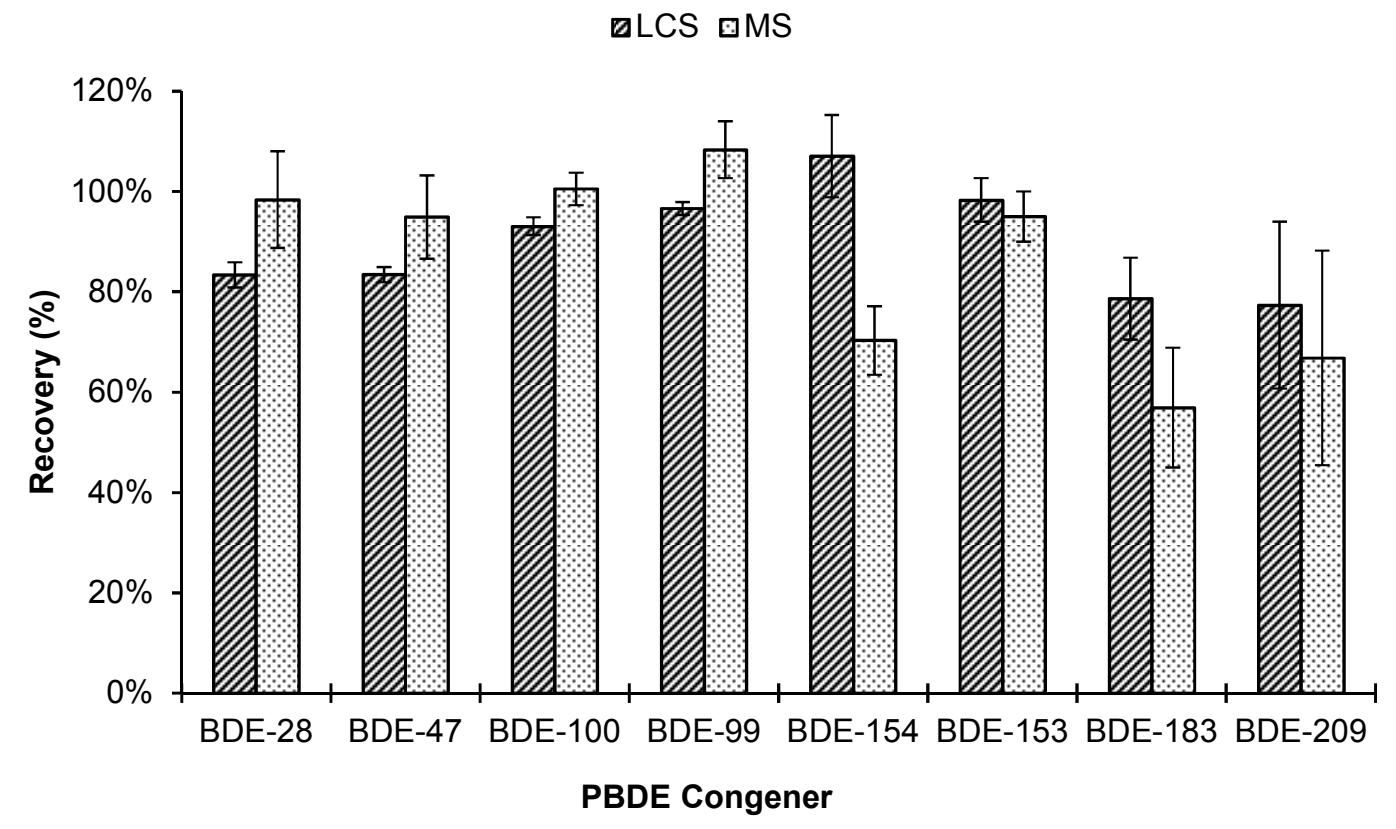

Figure S2. Recovery of LCS and MS samples analysed alongside experiment samples (mean $\pm 1 \mathrm{SE}, \mathrm{n}=3$ ). LCS and MS samples were spiked with $10 \mathrm{ng}$ of each target PBDE (100 ng of BDE-209). 